\title{
COPIM
}

\section{COPIM Experimental Publishing Workshop - Part 2: Promoting Experimental Publishing}

Sam Moore, Janneke Adema

Published on: Oct 20, 2020

DOI: $10.21428 / 785 a 6451 . a 21 d 57 b 6$

License: Creative Commons Attribution 4.0 International License (CC-BY 4.0). 


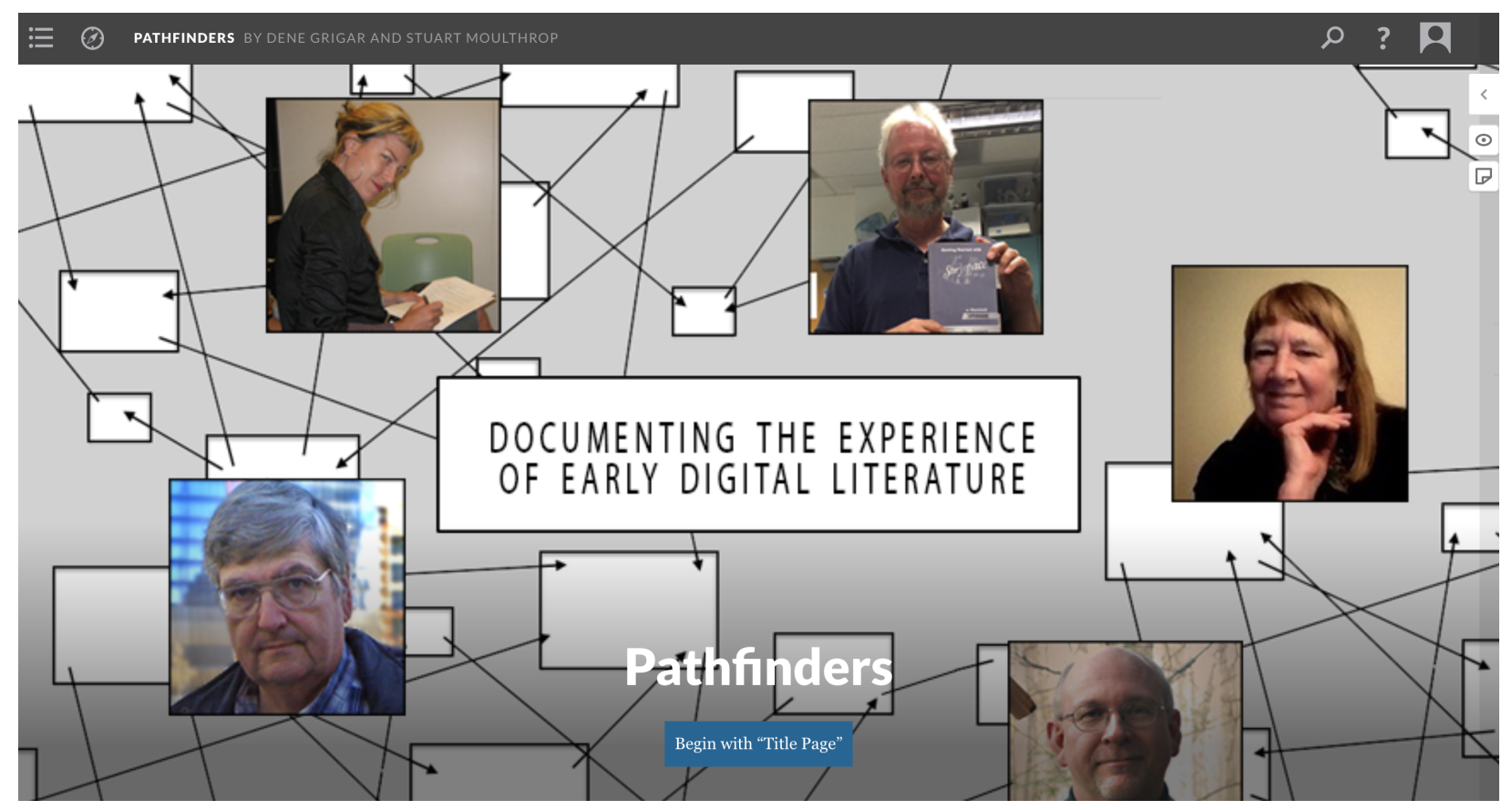

Landing page of Pathfinders by Dene Grigar and Stuart Moulthrop, a multimedia, open source book authored with Scalar. https://scalar.usc.edu/works/pathfinders/index

Following on from our previous post - summarising our discussion of inhibitions towards experimental publishing - this post looks at how we can stimulate experimentation, looking to understand how it can be encouraged within academic publishing and how some of the inhibitions described previously can be addressed. The following is a summary of our discussions.

Underlying our discussions were the following questions:

- How can we stimulate the uptake of experimental publishing and the creation of experimental longform publications, and the reuse of and engagement with OA books?

- What projects/platforms/software do we need to be aware of and in touch with?

- What strategies should we devise to stimulate experimentation and reuse?

\section{Rewarding experimentation}

To address the issue of institutional recognition that continues to plague more experimental forms of publishing our participants suggested various ways that we can start to recognise and reward experimentation, for example with the help of prizes or grants dedicated to publishing experiments. Andrew Lockett (Westminster University Press), suggested that elements of gamification could be one way of facilitating this where experimentation is 'the name of the game' and is rewarded as such.

Similarly, Whitney Trettien (thresholds) highlighted the power of prizes and providing grants and how this can help to confer prestige to certain kinds of activities, including experimental forms of publishing. These prizes could be tied to collaborations with scientific disciplines, museums, or 
funders, particularly around urgent issues such as climate change or new ways to connect humanities research to different audiences. For Tara McPherson (Scalar), academics might be more willing to experiment if there were common rewards structures for recognising the value of experimentation "beyond the intellectual," again referencing the double-work load, were experimentation is something that people do on top of their 'normal' requirements.

Related to this is the capacity for experimentation to make publishing fun and exciting again, rather than a largely uninterrogated practice to conform with. It therefore speaks to a broader need to reassess publishing practices and to understand new ways to motivate researchers more generally. For Andrew Lockett, publishing often connotes a sense of duty for the average academic and so experimentation would be one way of injecting a renewed sense of excitement into research dissemination, thus making it less of an obligation.

\section{Community Engagement During the Research Life Cycle}

One thing that became clear from our discussions was that experimental publishing benefits when authors and publishers already establish connections during the research process, instead of at the back end of it, which is more commonly the norm within academic book publishing. Both Alessandra Tosi (Open Book Publishers) and Heather Staines (Knowledge Futures Group/PubPub) described how publishers could adopt approaches that seek to show authors early on in the research process that their work would be suitable for publishing experimentally by illustrating the benefits of doing so. As Heather Staines explained: "It's a multi-faceted conversation that needs to happen between an editor and someone who knows technological capabilities and an author and maybe even beyond that, talking about what might be possible." As Sarah Kember (Goldsmiths Press) made clear, publishers could be "projecting a more expansive set of possibilities and permissions," giving people permission to do work a little bit outside of the box, as Goldsmiths Press sets out to do. Andreas Kirchner explained that Meson Press does this through engaging with learned societies from an early stage in order to draw authors into a project so they can "learn about these new possibilities" of experimentation, where "then they get drawn into a project" ... "and then it becomes more fun too for them when they see that something comes out of it." As such, as it often relates to the entire research life cycle of a project. Experimental publishing allows publishers to embed themselves and to better serve their author communities by reorienting publishing towards their situated practice. This relates to how experimental publishing is, for Adam Hyde (Coko), itself a "community process" rather than a mere service provided by publishers. Beyond reaching into other author communities throughout the course of the research process, participants also noted the potential of experimental publishing to build more community between publishers. This could be about sharing best practices, as Cheryl Ball (Vega) noted, in a way that makes fellow publishers collaborators rather than competitors. 


\section{Teach it!}

More pragmatically, many participants described the institutional basis for getting people involved with experimentation. Many of the academics at the workshop described the importance of both teaching experimental publishing practices and having students engage with experimental works themselves, which might involve, as Whitney Trettien explained "reaching out to instructors to teach the work and use it." Sarah Kember highlighted the gap between research and pedagogical materials, something that experimental publishing could bridge through its often-interactive nature. This could help get scholarship into the hands of a wider audience, especially through larger public-facing institutions such as MIT and Michigan. Andrew Lockett highlighted the library's role in this, particularly their ability to connect between academics and students, along with their increasing role in publishing itself. Cheryl Ball also noted that this was the main reason for her move from faculty to a library setting, so she could teach faculty members, graduates, and students how to do experimental forms of publishing within rhetoric and composition/writing studies.

Alongside this pedagogical component, participants explored the possibilities of encouraging experimental publishing by showing authors and publishers the available platforms, tools and technologies to create their work and which presses would be best suited to support them. As was discussed, this could take the form of a centralised organisation that advocates for experimental publishing (an Experimental Monograph Association, as Andrew Lockett suggested), or collaborations between different publishers and libraries where they recommend the services of one another (as is already happening within the scholar-led and university press ecosystem). For Julien McHardy (Mattering Press), a great deal of editorial work is devoted to simply explaining the possibilities of experimentation for a particular project to its author, work which might otherwise be shared across the experimental publishing ecosystem. While on the production side, Lara Speicher (UCL Press) noted that a common production system might be beneficial for publishers to tap into and deliver "bespoke" publishing services to authors. Cheryl Ball noted how there is a role here for summer camps and institutes too (she mentioned in the US the Vectors Institute, KairosCamp, the DMAC Institute, and

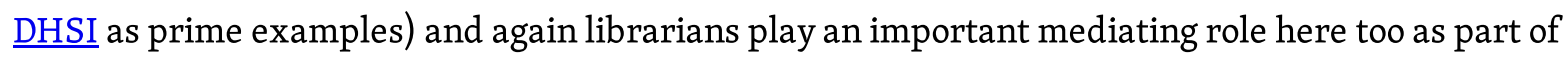
digital scholarship or publishing units, often situated within the library.

\section{Funding}

A lot of experimental publishing opportunities (as is the case with this work package and with COPIM, which was funded by Research England and Arcadia) have been made possible by funding. Within the US the Mellon Foundation has played a quintessential role in this, partly funding both Vega and Manifold for example. Whitney Trettien explained that what enabled her to complete her book was an "NEH/Mellon digital publications grant, which is basically a NEH fellowship but intended to give you time to focus on the digital portion or more experimental portion of the monograph. That was 
invaluable because I had only one semester leave pre-tenure through my university." Yet as Cheryl Ball outlined, a lot of experimental publishing projects (such as Kairos) rely entirely on volunteer effort and a lot of the more interesting experimental publishing work happens at "rogue schools," and not as might be expected at the well funded R1 research universities in the US. As Andreas Kirchner similarly stated, meson press works because it is small scale and niche, which can give the press freedom to experiment. As such Tara McPherson mentioned that US academic publishers might benefit more from doing what some European publishers and authors do: step outside and work alongside the university system to do this kind of work. It is also worth noting that, as Andrew Lockett identified, too much funding often creates the search for the "killer app" that often ends up rehearsing many of the conditions of print publishing. There is perhaps something to be said for the DIY nature of experimental scholarship that might best be supported through grassroots collaborations and other partnerships, although our participants were quick to point out that funding does remain essential in this landscape.

\section{Ethical, accessible, and open publishing cultures}

One final noteworthy theme that came up as part of our discussions is the possibility of encouraging experimentation through its foregrounding of ethical and accessible publishing cultures across the whole publication process. For example, participants explored the potential of experimental publications and infrastructures to promote translations of content. Simon Worthington (Hybrid Publishing Group) and Susan Doerr (Manifold) each discussed different software approaches to content translation, such as Public Knowledge Project's OJS software (OJS, OMP, and other PKP software applications are multilingual) and University of Minnesota's Manifold which ingests content in any Unicode 8 language. However, many user interfaces remain accessible in English only, so there are opportunities to further diversify and improve. Alongside linguistic accessibility, participants mentioned the fact that software for experimental publishing is often designed with reader accessibility in mind through digital reading software, dyslexia-friendly fonts and other accessibility tools , thus highlighting the advantages of experimental platforms over print and many other standardised forms of print publishing.

In a similar ethical vein, participants discussed the relationship between experimental publishing and community-control, open infrastructure and other ethical approaches to content dissemination and ownership. Sarah Kember described working with Heather Staines and MIT's Knowledge Futures Group who are thinking about the 'next move' beyond open content. For Kember, this requires a reassessment of the structures of knowledge across the university, something that experimental publishing can play a part in helping to determine. There is also, as many participants noted, a politics associated with experimental publishing that tends towards community ownership over commercial platforms. Because of its often indeterminate nature, it also offers an opportunity to explore 
potentially more ethical cultures across the publishing process, rather than just through formal publishing outputs.

Header image: "Pathfinders: Documenting the Experience of Early Digital Literature" by Stuart Moulthrop and Dene Grigar is licensed under a Creative Commons AttributionShareAlike 4.0 International License. Based on a work at http: //scalar.usc.edu/works/pathfinders. 